\title{
REVIEWS
}

\section{An Individualized Approach to Cancer Screening Decisions in Older Adults: A Multilevel Framework}

\author{
Erica S. Breslau, Ph.D., M.P.H. ${ }^{\text {}}$, Sherri Sheinfeld Gorin, Ph.D. ${ }^{2,3}$, \\ Heather M. Edwards, Ph.D., M.P.H., M.B.A. ${ }^{4}$, Mara A. Schonberg, M.D., M.P.H. ${ }^{5}$, \\ Nicole Saiontz, M.H.S. ${ }^{6}$, and Louise C. Walter, M.D. ${ }^{7}$ \\ 'Healthcare Delivery Research Program, Division of Cancer Control and Population Sciences, National Cancer Institute, Rockville, MD, USA; \\ ${ }^{2}$ Frederick National Laboratory for Cancer Research, Leidos Biomedical Research, Inc., Frederick, MD, USA; ${ }^{3}$ New York Physicians Against Cancer, \\ Herbert Inving Comprehensive Cancer Center, New York, NY, USA; ${ }^{4}$ Patient-Centered Outcomes Research Institute, Washington, DC, USA; \\ ${ }^{5}$ Department of Medicine, Division of General Medicine and Primary Care, Beth Israel Deaconess Medical Center, Harvard Medical School, \\ Boston, MA, USA; ${ }^{6}$ Office of the Director, Division of Cancer Control and Population Sciences, National Cancer Institute, Rockville, MD, USA; \\ ${ }^{7}$ Department of Medicine, Division of Geriatrics, University of California San Francisco and the San Francisco VA Medical Center, San Francisco, CA, \\ USA.
}

Guidelines for optimal cancer screening in older adults remain unclear, particularly for adults over the age of 75 . While cancer screening in older adults may benefit some in good health, it may cause unnecessary burdens in others with limited life expectancy. Thus, a systematic approach to enable individualized cancer screening decisions in older adults is needed. We suggest a framework that guides such decisions through evidence-based approaches from multiple interactions, and that involves the patient, clinician, and healthcare system. An individualized approach considers differences in disease risk rather than the chronological age of the patient. This paper presents a comprehensive framework that depicts the independent and converging levels of influences on individualized cancer screening decisions in older adults. This Individualized Decisions for Screening (IDS) framework recognizes the reality of these interrelationships, including the tensions that arise when behaviors and outcomes are valued differently at the patient, clinician, and healthcare organization levels. Person-centered approaches are essential to advancing multilevel research of individualized cancer screening decisions among older adults.

KEY WORDS: Individualized screening; Shared decision making; Geriatric; Multilevel; Framework.

J Gen Intern Med 31(5):539-47

DOI: $10.1007 / \mathrm{s} 11606-016-3629-\mathrm{y}$

(c) Society of General Internal Medicine 2016

\section{INTRODUCTION}

Age is a risk factor for most cancers. However, due to the paucity of published randomized clinical trial data for adults 75 and older, the risks and benefits of screening for many cancers are ill-defined. Health status among individuals in this age group varies widely, ranging from robust to frail. ${ }^{1}$ As this variance increases with age, cancer screening decisions become increasingly complex. ${ }^{2,3}$

Received October 9, 2015

Revised February 5, 2016

Accepted February 5, 2016

Published online March 3, 2016
Recent guidelines encourage an individualized decisionmaking approach among older adults, where the risks and benefits of screening tests are weighed and the quantity and quality of life are considered. ${ }^{1,3,4}$ These considerations are foundational to informing patient screening choices. ${ }^{5,6}$

In clinical practice, implementation of preference-sensitive cancer screening depends on factors at the patient, clinician, and healthcare organization levels. For example, patients may not make preference-informed medical decisions if clinicians do not engage them in discussion about the best evidence. ${ }^{5}$ Similarly, healthcare organizations may not incentivize such patient-clinician communication if patient loads and processes leave clinicians with little time or support to address the complexity of individualized decisions for older adults. ${ }^{7,8}$ The interconnected levels of the healthcare system call for a multilevel framework to improve research and communication regarding cancer screening in this age group. The framework has its genesis in the Patient Protection and Affordable Care Act and the Institute of Medicine's emphasis on efficient patient-centered care. These influential sources call for organizations to promote coordinated care across the cancer care continuum. ${ }^{5}$ The adoption of individualized decision making emphasizes important discussions between clinicians and patients regarding the delivery of care.

\section{NEW PARADIGM}

The Individualized Decisions for Screening (IDS) framework is intended to guide research aimed at improving tailored cancer screening decisions for adults over the age of 75 years, thereby reducing over-screening and under-screening among older adults (see Fig. 1). Individualized decisions are based on available scientific evidence, consider patient values, allow for discrete differences in disease risk, distinguish subsequent outcomes, are made by a well-informed patient, and are concordant with patient-specific preferences. ${ }^{5,6,9,10} \mathrm{We}$ use the term "individualized decisions" to indicate that decisions about cancer screening are a multifaceted process. ${ }^{5}$ 
The framework, graphically displayed in Figure 1, depicts the factors influencing variations in health outcomes at three distinct levels - patient, clinician, and healthcare organizatio$\mathrm{n}$ - and describes three outcome categories - proximal (e.g., transfer of information resulting from the quality of the decision process), intermediate (e.g., short-term outcomes of the decision), and distal (e.g., long-term outcomes of the decision) - that can be linked to individualized decision making. A unique characteristic of this framework - which is focused on adults age 75 and older - is the inclusion of the contextual factors influencing the outcomes resulting from an individualized decision to screen or not to screen. Outcomes are the ultimate indicator of the individualized decision process and screening performance. These factors and outcomes were determined from multiple quantitative and qualitative research studies grounded in communication and organizational theory. Individualized decision making was developed by Walter, ${ }^{4}$ patient-centered communication from the works of Epstein, ${ }^{11}$ and Roter, ${ }^{8}$ and organization theory from Shortell, ${ }^{12}$ and Wagner ${ }^{13}$. The intent of this paper is to present a framework that identifies multilevel gaps in evidence that must be addressed to improve individualized cancer screening decisions among older adults.

\section{Decision Factors at the Patient, Clinician, and Healthcare Organization Levels}

i. Patient factors that influence individualized decisions Previous screening experiences and habits, ${ }^{14,15}$ societal norms and pressure from family, ${ }^{14}$ literacy, ${ }^{16}$ risk perception, ${ }^{14,17}$ costs, ${ }^{18}$ and access to care and to screening tests ${ }^{19}$ are among the factors that influence patient decisions regarding cancer screening. ${ }^{20,21}$ Patient preferences and values are not always clear. ${ }^{22}$ For example, patients over the age of 80 who consider screening might place greater value on years of life with higher quality than on more years of life but with lower quality, and their preference not to be screened is very clearly defined. ${ }^{20,21}$ In addition, the desire to live longer might motivate screening, ${ }^{14}$ while preparation for the end of life might preclude cancer screening. ${ }^{14,15,17}$ In general, however, life expectancy does not seem to weigh heavily in patients' screening decisions. Related anxiety ${ }^{23,24}$ and the need for reassurance $^{14,17}$ are other psychosocial influences that affect patient's choices. ${ }^{15,24}$

ii. Clinician factors that influence individualized decisions In the United States, clinician recommendations, or lack thereof, have been identified as the strongest influence on patient screening decisions. ${ }^{1,20,25,26}$ To enhance favorable outcomes and reduce harm, clinicians must engage in informed discussion. ${ }^{5}$ Clinicians, more so than patients, tend to consider age, health, functional status, and life expectancy in screening decisions. ${ }^{1}$ For example, they may recommend screening for patients whom they perceive to be in good health, and recommend against screening - or choose not to discuss cancer screening with those in poor health. ${ }^{27}$ Clinicians may also be influenced by the patient's living situation and family support, ${ }^{21}$ the patient's personality and previous screening behavior, ${ }^{21}$ and the proximity to sites for cancer treatment. ${ }^{17,27}$ When clinicians are uncertain of the ratio of benefit to harm of cancer screening, ${ }^{17,28}$ decision making can be challenging. ${ }^{3,20,26,27}$ Discomfort in estimating and discussing the patient's life expectancy, lack of data on risks and benefits of cancer screening tests among older age groups, and conflicting guidelines impede individualized decisions. $^{5}$

Clinician-patient discussions regarding cancer screening are also influenced by the time available in a clinic visit $^{17,21}$ and the frequency of patient visits. ${ }^{21}$ Patients who frequently visit a clinician are more likely to be screened, regardless of their health. Clinicians feel most comfortable discussing life expectancy when they have a long-standing, trusting relationship with their patient. ${ }^{17}$

iii. Healthcare organization factors that influence individualized decisions

Inherent in any screening program are organizational structures, processes, norms, and values that support (or impede) clinical decision making. The prioritization of individualized decisions within an organization's culture plays an important role, even in small organizations. Cancer screening is influenced by practice size or type, resources or facilities for screening including on-site screening availability, staffing mix (ratio of generalists to specialists), organizational culture (perceived commitment to service quality, education programs), quality measures, and cost. ${ }^{29,30}$

\section{Three Categories of Outcomes: Proximal, Intermediate, and Distal}

Cancer screening outcomes occur over various time horizons that may be valued differently at the patient, clinician, and healthcare organization levels. Studies examining the relationship between individualized decisions and health outcomes are lacking among older adults. ${ }^{2}$ Proximal outcomes occur immediately, and depend on the quality of the screening decision process itself (e.g., improved patient knowledge of choices). Intermediate outcomes are effects seen shortly after the receipt of screening or the decision not to screen, and include possible complications of a screening test or treatment, or relief from avoiding medical interventions with unproven benefit. Distal outcomes describe the longer-term impacts of the individualized decision process. For example, the decision to screen can lead to earlier diagnosis and treatment, which can maximize individual health, reduce mortality, and minimize sequelae from treatment that can affect quality of life..$^{29,30}$

It is important to note that patients, clinicians, and organizations interact to produce a set of medical decisions (e.g., test 


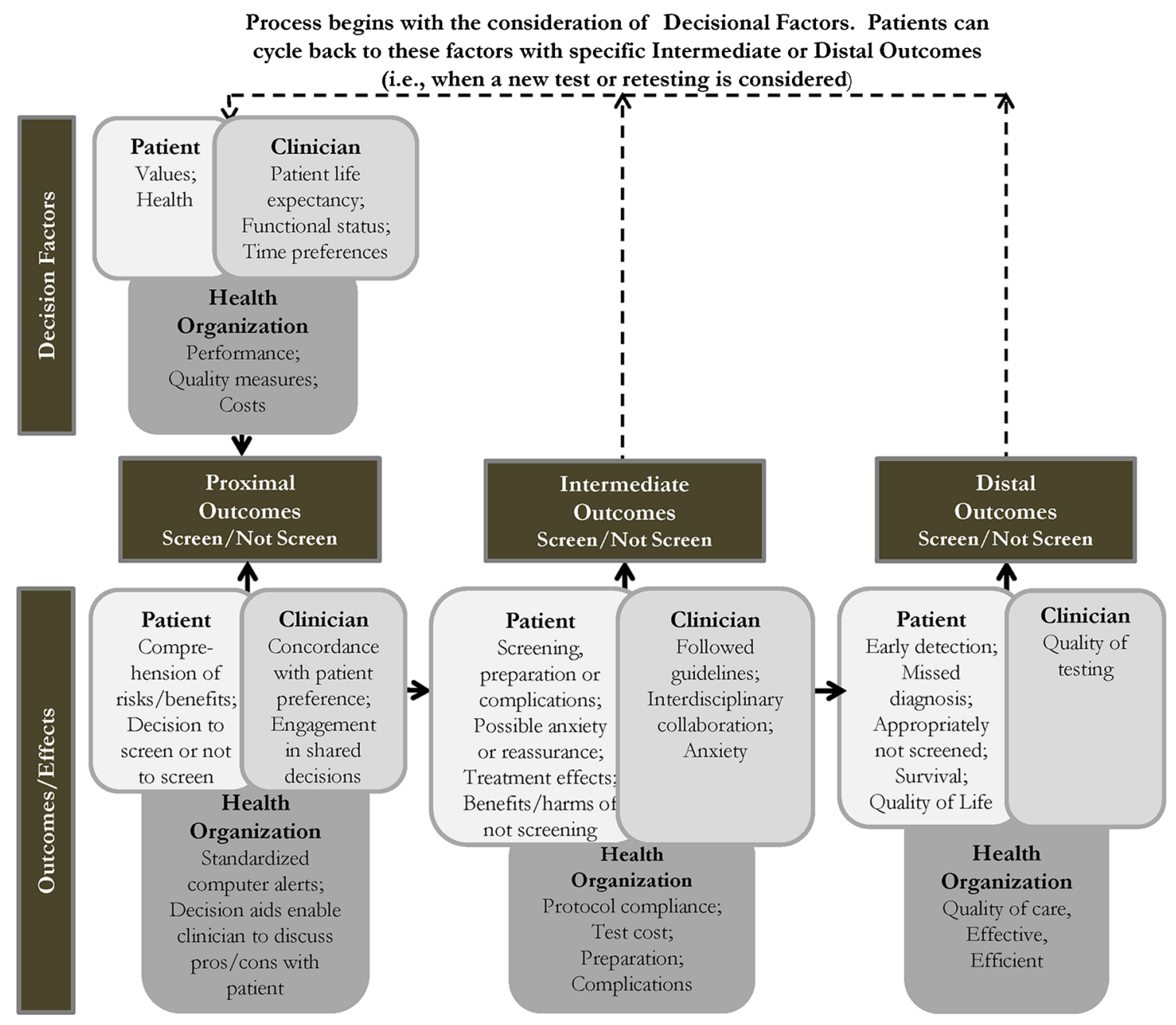

Figure 1. Individualized Decisions for Screening of Older Adults (IDS). Arrows indicate the direction of causality. Solid lines denote the link between outcomes resulting from an individualized decision and a link to an outcome stemming from the patient, clinician, and healthcare organization. Dotted lines specify an intermediate or distal outcome whereby individual patients cycle back to Decisional Factors should a new test or retesting be ordered by a clinician.

ordering, referral, follow-up visits, treatment, no screening, etc.) that will affect the patient's health outcomes. However, the decision outcome - and ultimately, health outcomes - may be modified by all or any combination of patient, clinician, and organization. For instance, patients may refuse to be tested or receive treatment, insurance companies may deny payment for diagnostic tests or treatment, or the screening tests might not be available or not ordered. ${ }^{30}$

Examples of each outcome category are described below.

i. Proximal Outcomes (attributes of the decision process to screen or not to screen)

At the proximal level, an individualized decision is associated with clinician communication, patient awareness of pros and cons, and the engagement of both in shared decision making. ${ }^{2,5,8,21}$ Patients may focus on aspects of the decision that are important to them, clinicians may focus on screening decisions in concordance with patient preferences and with guidelines, and the healthcare organization may be concerned with the delivery of personcentered high-value care. ${ }^{8}$
Patients. Even though patients bring their own health values to the decision process, their ability to express their values as a screening preference, especially for mitigating uncertainty, is important. In order to engage in behavioral self-management, the patient must have an understanding of all the benefits and risks of testing, which is gained through being actively informed by the decision process with the healthcare professional. ${ }^{2}$ In addition to measuring knowledge, assessing whether questions were answered and information was tailored to patient needs - especially those with low literacy or who are socially disadvantaged - and determining patient satisfaction with the outcome provides insight into the quality of the screening decision. ${ }^{5,11,20}$

Clinicians. In addition to presenting the patient with facts about the benefits and drawbacks of screening, clinicians facilitate an individualized decision-making process by reaching concordance with the patient's preferences. Assessing the quantity and quality of information required to educate patients about how their personal health values may affect 
screening decisions can provide insight into the shared communication process. ${ }^{20}$ Similarly, understanding how carefully clinicians listen to patients and offer information about the risks and impact of screening options, including uncertainties and tests that could be harmful, unnecessary or costly, can provide insight into the quality of the discussion. ${ }^{5,30}$ Different interpersonal and communication styles can also affect outcomes. For example, some individualized discussions may require clinicians to act as a counselor and to engage patients in a joint process of understanding, by articulating values and suggesting relevant options such that patients are able to make a decision. Alternatively, a clinician in a role of guardian articulates the value on behalf of the patient. Such decisions may be independent of the patient's preferences, which may be unknown or unarticulated. ${ }^{30}$

Organizations. At the proximal level, healthcare organizations provide the system-level infrastructure for the delivery of person-centered care for patients and care coordination support to clinicians. ${ }^{16}$ Organizational resources and tools to encourage, empower, and motivate clinicians to adopt the practice of individualized decision making and to ensure sustained awareness of the decision-making process can range from the simple to the complex. ${ }^{5}$ Organizations are encouraged to measure decision quality with basic computergenerated alert programs (or decision aids) that could help prompt clinicians to engage in decision-making dialogues or with more sophisticated decision programs which incorporate patient-level characteristics to model likely outcomes of screening for a specific patient. ${ }^{5,31}$ Similarly, in response to external accountability demands or internal quality improvement initiatives, organizations may assess conflicts or inconsistencies within the context of health services delivery, particularly those arising between clinicians and patients regarding screening. ${ }^{9,20,29,30}$

ii. Intermediate Outcomes (of the decision to screen or not to screen)

Intermediate outcomes associated with an individualized decision are the effects seen shortly after receipt of screening or the decision not to screen.

Patients. Benefits of screening in older adults include patient satisfaction $^{32}$ and improved self-esteem. ${ }^{14,24}$ Cancer screening, however, may also cause patient harms, including pain and anxiety during and/or in preparation for the test (e.g., bowel preparation for colonoscopy), ${ }^{33}$ complications from the screening test itself (e.g., hospitalization for dehydration, bleeding, perforation after a colonoscopy) or from diagnostic tests following an abnormal finding (e.g., breast biopsy, removal of precursor lesion or bronchoscopy),${ }^{34}$ reassurance from false-negative tests, ${ }^{35}$ and overdiagnosis of tumors that are of no threat. ${ }^{33}$ All complications may increase with age. $^{33,35}$

Clinician. Outcomes are tied to whether the decision is in keeping with current guidelines, which are currently limited to age-based recommendations for breast and colon cancers. ${ }^{1,3}$ The United States Preventive Services Task Force (USPSTF) applies a rating system in which the strength of the evidence and the balance of benefits and harms are reflected in its recommendations. ${ }^{3,36-38}$ The evidence for health outcomes in older patients is relatively weak, however, as older individuals and those with comorbidities are rarely included in clinical trials. ${ }^{20}$ Clinicians and patients must thus decide the best screening approach during wellness and chronic disease management visits. ${ }^{3}$ Clinicians do report anxiety when dealing with an older age group, especially should a patient appropriately choose not to be screened and later be diagnosed with cancer. $^{24}$

Organizations. Fee-for-service healthcare organizations generally benefit financially from increased test use. Therefore, organizations may monitor the healthcare delivery process for costs, such as those associated with preparations for screening, for diagnostic follow-up and treatment for cancer, and for test error or overuse. To improve intermediate outcomes, organizations that advance individualized decisions as a central tenet must work closely with clinicians to incorporate clinical findings with the progress of groups of patients over time. Organizations will need to couple the measurement of existing electronic reminders and decision aids with new mobile technology advancements that encourage and measure "meaningful choices" from patients. ${ }^{5,29}$ More sophisticated electronic health records (EHRs) have the potential to identify variations in service utilization across population groups. ${ }^{39}$

iii. Distal Outcomes (of the decision to screen or not to screen)

The longer-term consequences of the individualized decision will be best assessed by following patients over time, with standardized outcome measures to determine the patient, clinician and organizational factors that contribute across cancer organizations. The framework notes the importance of improved survival as a primary distal outcome for patients, clinicians and healthcare organizations alike. Together with the second outcome of improving quality of life, this will require collecting data from multiple sectors, including private and public, to monitor whether the health indicator is being met.

Patients. There is a pressing need for distal outcome data in older adults. A meta-analysis of randomized controlled trials of cancer screening of adults up to age 75 years found that, on 
Table 1 Recommended Directions for Future Research to Improve Individualized Cancer Screening Decisions Across Patient, Clinician and Healthcare Organization Levels

\begin{tabular}{lll}
\hline \hline Outcome Examples & Patient & Clinician \\
\hline Proximal Outcomes & $\begin{array}{l}\text { Depends on patient's participation in an engaged communication, clinician's clear explanation and organizational } \\
\text { support }\end{array}$
\end{tabular}

Ensure individualized decisions are made with quality discussions of risk that close the gap between the patient, clinician and organization. For example, potential risks associated with screening require conversations with patients since risk/benefit ratios change with diminished health and decreased life expectancy

Coordinate the processes through which individualized decisions are communicated during clinical interactions

Develop options that address conflicting screening priorities

\section{Intermediate Outcomes}

Support organizational involvement in quality-of-care outcomes (i.e., timely and equitable access to screening, diagnostic testing and/or specialty care) and coordinate that care across different healthcare systems

Identify and report poor outcomes resulting from screening

Develop novel methods that balance individualized decision interactions between and across healthcare levels

Support active, involved patients who participate in shared decision making about their healthcare. For example, studies that determine whether patients are truly informed about the risks and benefits after a discussion; measuring whether patients are adequately informed and are cognizant of their values

Studies that identify how older patients engage in new technologies that encourage individualized shared decisions

Identify and monitor whether older, sick patients with an interest in screening understand harms of screening

Studies that engage patients in conversation about their health. Offers options based on medical evidence so that patients make informed decisions based on benefits and risks of screening. Need to understand what clinician training is necessary to enable them to have conversations about patient life expectancy in the setting of cancer screening decisions

Strategies for improving clinician education and training to remain up to date on emerging evidence, professional guidelines, and support of individualized decisions and communication with patients

Interventions that facilitate the decision-making process with the management of uncertainty, and promote the benefits and risks of screening in older adults according to life expectancy
Creates a supportive environment (i.e., educational and problemsolving resources) for clinicians and patients. Studies that develop and provide tools for individualized decisions to take place, and that recommend methods to reward individualized care
Identify pathways through which contexts directly and indirectly affect the development of normative influences, structures and technologies to promote clinician-patient interactions leading to individualized decisions

Develop electronic tools to align patient-clinician perspective and encourage patient-focused care

Results from patient and clinician individualized decision (i.e., test ordering/not ordering, referral, follow-up visit, treatment, reassurance, no screening) will affect health outcomes for the patients

As people age, determine how best to deliver instructions for test preparation and test novel (technology) reporting modalities so that older patents know how to report complications in a timely manner

Differentiate technologies that are acceptable to older patients so that they are more likely to understand ahead of a clinical conversation which tests and treatment outcomes may result in possible harms

Encourage research that uses novel methods for tracking and implementing decision outcomes with diverse racial-ethnic, socioeconomic and geographic groups of older adults
Develop technology processes that adapt to evolving guidelines; manage the needs of older patient to simplify the referral and access to specialists

Develop new channels for reporting on adverse test outcomes that are easy and acceptable for clinicians

Identify optimal approaches to provide better understanding of electronic decision tools to assist in interactions with older patients
Develop procedures tailored to older adults that monitor patientcentered quality of care, coordination of care, and all outcomes (complications) from test ordering. Devise systems to coordinate care across different clinical offices and healthcare systems

Determine how best to promote widespread adoption and implementation of protocols that report on test complications resulting from screening and document the uncertainty of screening benefit

Develop methods that support an organization's need to track progress towards achieving clinical goals (balanced decisions).

Determine how to maximize patterns of healthcare delivered (functional status, pain, satisfaction, and cost) between and across healthcare levels that result from screening decision outcomes average, it takes 10 years before one death from breast and colorectal cancers are prevented for every 1000 patients screened. ${ }^{1,40,41}$ These results suggest that there might be a minimum life expectancy of 10 years to see any mortality benefit from these cancer screening tests. ${ }^{1,41}$
In addition to a potential mortality benefit, early detection can reduce the need for more aggressive care and the accompanying declines in health-related quality of life. ${ }^{26,42}$ Also, when results are accurately negative, screening can have a positive distal outcome by providing long-term reassurance to older adults 
Table 1. (continued)

\begin{tabular}{|c|c|c|c|}
\hline Outcome Examples & Patient & Clinician & Healthcare System \\
\hline Distal Outcomes & \multicolumn{3}{|c|}{ Long-term consequence of the individualized screening decision to screen or not to screen } \\
\hline $\begin{array}{l}\text { Perceptions of optimal health and } \\
\text { quality decisions are subjective. } \\
\text { Understand the outcome for each } \\
\text { outcome level with the use of } \\
\text { definitive objective endpoints such } \\
\text { as health efficiency (decreasing } \\
\text { healthcare use and expenditures) } \\
\text { and mortality }\end{array}$ & Patient quality of life & Clinician quality of testing & $\begin{array}{l}\text { Healthcare organization quality of } \\
\text { care }\end{array}$ \\
\hline $\begin{array}{l}\text { To be more patient-centered, } \\
\text { intervention studies need to include } \\
\text { the direct measurement of patient } \\
\text { well-being, and guidelines should } \\
\text { be patient-centered. Measures of } \\
\text { big data need to integrate patient, } \\
\text { clinical, administrative and } \\
\text { population data so that proximal } \\
\text { measures may be linked to distal } \\
\text { outcomes }\end{array}$ & Patient well-being & Improves patient safety & $\begin{array}{l}\text { Administrative databases, cancer } \\
\text { registries, electronic medical } \\
\text { records }\end{array}$ \\
\hline $\begin{array}{l}\text { Explain underlying modifiable } \\
\text { communication factors in } \\
\text { individualized decisions that } \\
\text { account for some of the observed } \\
\text { differences in outcomes }\end{array}$ & $\begin{array}{l}\text { Role and function of family and/or } \\
\text { social support of patient }\end{array}$ & Quality of medical decisions & $\begin{array}{l}\text { Continually adjusts to new research } \\
\text { and practice }\end{array}$ \\
\hline
\end{tabular}

about their health. ${ }^{18}$ Adults appropriately not screened (or who thus avoid the diagnosis of a cancer that would not lead to morbidity or mortality) may have improved quality of life by avoiding overdetection and subsequent overtreatment of cancer.

Clinician. Optimal patient outcomes may affect a clinician's professional evaluation, satisfaction, and overall financial remuneration. Conversely, the clinician's liability as a result of a missed diagnosis due to failure to conduct a recommended screening could have deleterious effects. ${ }^{43}$

Organization. Distal outcomes at the healthcare level are many. First, in the event of a positive test, the diagnosis and treatment of cancer are costly, especially among patients with multiple chronic problems. ${ }^{43}$ Second, population-based measures of access, uptake of preventive services, and mortality rates may be used by organizations to assess individualized decisions and the quality of the healthcare organization. ${ }^{44}$ Existing organizational performance indicators about cancer screening assess clinic waiting times, standards of safety such as infection rates, unintended consequences of tests, and unexpected deaths from errors.

Third, even though administrative data were never intended for use in quality assessments, meaningful indicators can be derived from these data. ${ }^{45}$ At present, performance ratings measure the outcomes rather than the processes of care in cancer screening decisions. ${ }^{46}$ Thus existing performance measures for the organization may fail to capture, monitor, and reward individualized decisions. ${ }^{46}$ Using the IDS framework, however, healthcare organizations could provide clinicians with individualized rates of screening by patient health category (using the
Charlson Comorbidity Index). ${ }^{1,3,4}$ Ideally, clinicians would see high screening rates among those in good health and low screening rates among those in poor health. Healthcare claims datasets linked to individualized decisions between clinicians and patients, together with organizational quality surveys of patient well-being, could provide a feedback loop among distal outcome measures, patients, clinicians and organizations.

\section{Interrelationships at the Patient, Clinician, and Healthcare Levels}

Each level influences the other, either implicitly or explicitly. For example, patients and clinicians are never really isolated from the healthcare organization in which they are embedded, even in small clinician-led offices. ${ }^{47}$

In order for patients to make an individualized decision regarding screening, clinicians must provide them with information about the potential benefits and risks of screening. Studies suggest that few older adults are adequately provided with this information. ${ }^{17,48}$ In many cases, the benefits of screening are overestimated, and the harms of screening are underestimated or not discussed. ${ }^{43}$ Nearly all patients in one study reported that their clinicians addressed the benefits of cancer screening, but only around one quarter reported that they received information about the risks. ${ }^{43}$ Furthermore, evidence suggests that even among patients who prefer a passive role in medical decisions, ${ }^{41,49-51}$ many still want to be informed and have their values considered., ${ }^{4,51}$

At the healthcare organization level, the individualized decision-making process necessitates close collaboration 
between clinicians and healthcare organizations to ensure timely and appropriate follow-up care. ${ }^{29,52}$ In some instances, this requires the interdisciplinary collaboration of primary care clinicians, radiologists, surgeons, or pathologists to ensure necessary diagnostic work-up, intervention, or follow-up care. ${ }^{29,52}$ The evidence for patient-centered cancer care coordination by patient navigators is strong. Navigators integrate the shared, common interests of patients, clinicians, and healthcare organizations. ${ }^{53}$ In other instances, the individualized decision will require monitoring of decisions not to screen, or re-engaging the patient and clinician in screening discussions at a later time.

Healthcare organizations might also create a place in the medical record where clinicians can see that a patient decided to cease screening, so that patients would no longer receive mail reminders from radiology departments regarding screening. Healthcare organizations must monitor services delivered and patient complications so that these decision-making and coordination processes are continually refined and streamlined.

\section{DISCUSSION AND RECOMMENDATIONS}

The goal of this paper is to guide research with a multilevel framework, the IDS, to improve individualized cancer screening decisions in older adults. This framework is novel: it synthesizes the influences of individualized cancer screening decisions among older adults from patients themselves and from clinicians and healthcare organizations. Systems science may help determine which combination of decisions has the potential to contribute to broader change among specific older populations, such as when and when not to advise a screening test. The IDS suggests that decisions are linked to values at each level, and that considering multiple patient and population outcomes over time offers a different perspective on individual screening decisions. Our interest was in understanding how best to address the complexities of a screening decision across various levels of the healthcare system.

\section{FUTURE RESEARCH DIRECTIONS}

The IDS framework illustrates that to enhance the ability of patients, clinicians, and organizations to pursue individualized decisions, future research must address two key issues. First, an expanded evidence base must be developed that links patient characteristics such as age, functioning, comorbidities, and life expectancy with cancer screening decisions and with proximal, intermediate, and distal outcomes. To ensure that evidence is patient-centered, patient characteristics and test results can be embedded within the organizations where screening takes place. Similarly, improved electronic decision support tools for clinicians can facilitate interactions with patients who may not have clear values regarding health outcomes. To improve quality of care and reduce variation across practices, evidence-adaptive decision support systems can continually adjust to new research and practice. Such systems are ideal for risk-averse clinicians who may choose to recommend screening to adults based on age, regardless of the patients' health, due to concerns of litigation for a missed cancer diagnosis.

An expanded evidence base will begin to provide data that can contribute to systemic, individualized decisions. ${ }^{45}$ To engage in individualized decision making, clinicians must be trained in patient-centered communication and shared decision making, as they are then more likely to adjust their communication approach to enable decisions that are consistent with patients' needs, preferences, and values. ${ }^{8,20,51}$ Therefore, with additional training (i.e., academic detailing tailored to clinician barriers), clinicians may be able to lead balanced discussions around cancer screening for older adults. ${ }^{47,53}$ To facilitate shared decision making and reduce decisional conflict, on-line toolkits are available to help clinicians estimate patient prognosis and guide discussions about cancer screening (see www.eprognosis.org). ${ }^{54}$ Similarly, institutions that promote individualized medical decisions might make individualized decision aids routinely accessible to patients and families in order to enhance more "meaningful choices." Learning systems with data that extract patient-reported measures and have transitioned to Accountable Care Organization models within Medicare and Medicaid bring with them different emphases on prevention and patient lists.

The second key area of research surrounds the conceptualization, measurement, and implementation of multilevel interventions. The ability to effect change in the quality of cancer screening outcomes will be influenced both directly and indirectly by the interaction between patients, clinicians, and organizations. All have distinct goals. Interventions to advance individual knowledge, attitudes, and behavior must be tested at the patient, clinician, and organization levels to assess independent and synergistic effects. ${ }^{46}$ Interventions that simultaneously monitor organizational influences over time from the patient and clinician perspective will greatly reduce the gaps in standards of care. Clinical support systems that measure the timeliness of care, delivery of screening results, scheduling of diagnostic testing, safety of testing in order to avoid side effects, and patient-centeredness of care may be generalized to other clinical areas.

Importantly, the IDS framework can be modified if data from clinical trials or precision medicine research bring new understanding to factors that affect individualized screening decisions or their outcomes. See Table 1 for recommendations and examples about future directions of multilevel research focused on individualized decisions in older adults.

\section{CONCLUSION}

The new healthcare environment of the Affordable Care Act has brought increased momentum toward an understanding of the interrelated systems that contribute to the health and well-being of our older populations. Research that explores the multiple determinants that influence individualized decisions must undergo a paradigm shift, moving away from considering patient, 
clinician, or organization factors in isolation or at a single point in time, and instead addressing how patient, clinician, and organization factors together contribute to upstream proximal, intermediate, and distal outcomes following a screening decision. The IDS framework provides a guide to aid in measuring, tracking, and ultimately encouraging individualized decisions for cancer screening. Implementation of the framework and recommendations will require leadership to embolden patients, clinicians, and organizations to work together to improve the health of older populations.

Acknowledgments: Disclaimer for Drs. Edwards and Sheinfeld Gorin: This project has been funded in whole or in part with federal funds from the National Cancer Institute, National Institutes of Health, under Contract No. HHSN261200800001E (HME). Dr. Walter was funded by the National Institute on Aging, National Institutes of Health under grant number K24AG041180. The content of this publication does not necessarily reflect the views or policies of the Department of Health and Human Services, nor does the mention of trade names, commercial products, or organizations imply endorsement by the U.S. government.

Corresponding Author: Erica S. Breslau, Ph.D., M.P.H.; Healthcare Delivery Research Program, Division of Cancer Control and Population SciencesNational Cancer Institute, 9609 Medical Center Drive, Rockville, MD 20850-9761, USA (e-mail: breslaue@mail.nih.gov).

\section{Compliance with Ethical Standards:}

Conflict of Interest: Drs. Breslau, Schonberg, and Walter and Ms. Saiontz do not have a conflict of interest with consultancies, employment, honoraria, stock ownership, expert testimony, grants, patents or royalties. Drs. Edwards and Sheinfeld Gorin were employed by Leidos Biomedical Research, Inc., as contractors supporting the National Cancer Institute. Dr. Edwards is currently employed by the PatientCentered Outcomes Research Institute and Dr. Sheinfeld Gorin by New York Physicians against Cancer, Herbert Irving Comprehensive Cancer Center, New York, NY.

\section{REFERENCES}

1. Schonberg MA, Breslau ES, McCarthy EP. Targeting of mammography screening according to life expectancy in women aged 75 and older. J Am Geriatr Soc. 2013;61(3):388-95.

2. Street RL, Makoul G, Arora NK, Epstein RM. How does communication heal? Pathways linking clinician-patient communication to health outcomes. Patient Educ Couns. 2009;74(3):295-301.

3. Eckstrom E, Feeny DH, Walter LC, Perdue LA, Whitlock EP. Individualizing cancer screening in older adults: a narrative review and framework for future research. J Gen Intern Med. 2013;28(2):292-8.

4. Walter LC, Covinsky KE. Cancer screening in elderly patients: a framework for individualized decision making. JAMA. 2001;285(21):2750-6.

5. Alston C, Paget L, Halvorson G, et al. Communicating with patients on health care evidence. Washington: Institute of Medicine; 2012.

6. Jepson RG, Forbes CA, Sowden AJ, Lewis RA. Increasing informed uptake and non-uptake of screening: evidence from a systematic review. Health Expect. 2001;4(2):116-30.

7. Tinetti ME, Fried TR, Boyd CM. Designing healthcare for the most common chronic condition-multimorbidity. JAMA. 2012;307(23):2493-4.

8. Roter D, Hall JA. Doctors talking with patients/patients talking with doctors: improving communication in medical visits, vol. 2. Westport: Praeger; 2006.

9. Sepucha KR, Matlock DD, Wills CE, et al. "It's valid and reliable" is not enough critical appraisal of reporting of measures in trials evaluating patient decision aids. Med Dec Making. 2014;0272989X14528381.

10. Leavitt MO, Downing GJ. Toward a future of personalized cancer care. Cancer Am Cancer Soc. 2008;113(S7): 1724-7.
11. Epstein RM, Street RL Jr. Patient-centered communication in cancer care: promoting healing and reducing suffering. National Cancer Institute, NIH Pub No. 07-6225; 2007 Bethesda, MD 2007

12. Shortell SM, Kaluzny AD. Organization theory and health services management. In: Shortell SM, Kaluzny AD, eds. Health care management: organization design and behavior. 3rd ed. Albany: Delmar; 1994:17-43.

13. Wagner EH, Austin BT, Davis C, Hindmarsh M, Schaefer J, Bonomi A. Improving chronic illness care: translating evidence into action. Health Aff. 2001;20(6):64-78.

14. Elwyn G, Elwyn B, Miron-Shatz T. Measuring 'decision quality': Irresolvable difficulties and an alternative proposal. 2nd ed. New York: Oxford University Press Inc.; 2009.

15. Torke AM, Schwartz PH, Holtz LR, Montz K, Sachs GA. Older adults and forgoing cancer screening: "I think it would be strange." JAMA Intern Med. 2013;173(7):526-31

16. Brach C, Keller D, Hernandez LM, et al. Ten attributes of health literate health care organizations. Washington: Institute of Medicine of the National Academies; 2012.

17. Jepson RG, Hewison J, Thompson AG, Weller D. How should we measure informed choice? The case of cancer screening. J Med Ethics. 2005;31(4): 192-6.

18. Swan JS, Fryback DG, Lawrence WF, Sainfort F, Hagenauer ME, Heisey DM. A time-tradeoff method for cost-effectiveness models applied to radiology. Med Decis Mak. 2000;20(1):79-88.

19. Moher D, Schachter HM, Mamaladze V, Lewin G, Paszat L, Verma S, DeGrasse C, Graham I, Brouwers M, Sampson M, Morrison A, Zhang L, O'Blenis P, Garrity C. Measuring the quality of breast cancer care in women. Summary, Evidence Report/Technology Assessment No. 105. (Prepared by the University of Ottawa Evidence-based Practice Center under Contract No. 290-02-0021.) AHRQ Publication No.04-E030-1. Rockville, MD: Agency for Healthcare Research and Quality. September 2004.

20. Schonberg MA, Ramanan RA, McCarthy EP, Marcantonio ER. Decision making and counseling around mammography screening for women aged 80 or older. J Gen Intern Med. 2006;21(9):979-85.

21. Fagerlin APM, Abhyankar P, Col N, Feldman-Stewart D, Gavaruzzi T, Kryworuchko J, Levin CA, Pieterse AH, Reyna V, Stiggelbout A, Scherer LD, Wills C, Witteman HO. Clarifying values: an updated review. BMC Med Inform Decis Mak. 2013;13(Suppl 2):58.

22. Coulter A. Partnerships with patients: the pros and cons of shared clinical decision-making. J Health Serv Res. 1997;2(2):112-21.

23. Mathieu E, Barratt AL, McGeechan K, Davey HM, Howard K, Houssami N. Helping women make choices about mammography screening: an online randomized trial of a decision aid for 40-year-old women. Patient Edus Couns. 2010;81(1):63-72.

24. Salz T, Richman AR, Brewer NT. Meta-analyses of the effect of falsepositive mammograms on generic and specific psychosocial outcomes. Psycho-Oncology. 2010;19(10):1026-34.

25. Klabunde CN, Frame PS, Meadow A, Jones E, Nadel M, Vernon Sw. A national survey of primary care physicians' colorectal cancer screening recommendations and practices. Prev Med. 2003;36(3):352-62.

26. Schonberg MA, McCarthy EP, Davis RB, Phillips RS, Hamel MB. Breast cancer screening in women aged 80 and older: results from a national survey. J Am Geriatr Soc. 2004;52(10):1688-95.

27. Fox J, Zikmund-Fisher BJ, Gross CP. Older patient experiences in the mammography decision-making process. Arch Intern Med. 2012;172(1):62-4.

28. Elmore JG, Kramer BS. Breast cancer screening: toward informed decisions. JAMA. 2014;311(13):1298-9.

29. Yano EM, Soban LM, Parkerton PH, Etzioni DA. Primary care practice organization influences colorectal cancer screening performance. J Health Serv Res. 2007;42(3p1):1130-49.

30. Gerrity MS, DeVellis RF, Earp JA. Physicians' reactions to uncertainty in patient care: a new measure and new insights. Med Care. 1990;28(8):72436.

31. Bernabeo E, Holmboe ES. Patients, providers, and systems need to acquire a specific set of competencies to achieve truly patient-centered care. Health Aff. 2013;32(2):250-8.

32. Miles A, Rainbow $\mathbf{S}$, von Wagner $\mathbf{C}$. Cancer fatalism and poor self-rated health mediate the association between socioeconomic status and uptake of colorectal cancer screening in England. Cancer Epidemiol Biomark. 2011;20(10):2132-40.

33. Elmore JG, Harris RP. The harms and benefits of modern screening mammography. BMJ. 2014;348:g3824.

34. Elmore JG, Fletcher SW. Overdiagnosis in breast cancer screening: time to tackle an underappreciated harm. Ann Intern Med. 2012;156(7):536-7. 
35. Ogden LL, Richards CL, Shenson D. Clinical preventive services for older adults: the interface between personal healthcare and public health services. Am J Public Health. 2012;102(3):419-25.

36. Nelson HD, Tyne K, Naik A, Bougatsos C, Chan BK, Humphrey L. Screening for breast cancer: an update for the US Preventive Services Task Force. Ann Intern Med. 2009; 151(10):727-37.

37. Calonge N, Petitti DB, DeWitt TG, et al. Screening for colorectal cancer: US Preventive Services Task Force recommendation statement. Ann Intern Med. 2008;149(9):627-37.

38. Moyer VA. Screening for prostate cancer: US Preventive Services Task Force recommendation statement. Ann Intern Med. 2012;157(2):12034.

39. Krist AH, Glenn BA, Glasgow RE, et al. Designing a valid randomized pragmatic primary care implementation trial: the my own health report (MOHR) project. Implement Sci. 2013;8(1):1-13.

40. Gross CP, Soulos PR, Ross JS, et al. Assessing the impact of screening colonoscopy on mortality in the Medicare population. J Gen Intern Med. 2011;26(12):1441-9.

41. Gross CP, McAvay GJ, Krumholz HM, Paltiel AD, Bhasin D, Tinetti ME. The effect of age and chronic illness on life expectancy after a diagnosis of colorectal cancer: implications for screening. Ann Intern Med. 2006; 145(9):646-53.

42. Walter LC, Lindquist $\mathbf{K}$, Covinsky KE. Relationship between health status and use of screening mammography and Papanicolaou smears among women older than 70 years of age. Ann Intern Med. 2004; 140(9):681-8.

43. Harris RP, Sheridan SL, Lewis CL, et al. The harms of screening: a proposed taxonomy and application to lung cancer screening. JAMA Int Med. 2014;174(2):281-6.
44. Scott A, Sivey P, Ait Ouakrim D, et al. The effect of financial incentives on the quality of healthcare provided by primary care physicians. Cochrane Database Syst Rev. 2011;9(9):CD008451.

45. Baxter NN, Sutradhar R, Forbes SS, Paszat LF, Saskin R, Rabeneck L. Analysis of administrative data finds endoscopist quality measures associated with postcolonoscopy colorectal cancer. Gastroenterology. 2011;140(1):65-72.

46. Cleary PD, Gross CP, Zaslavsky AM, Taplin SH. Multilevel interventions: study design and analysis issues. JNCI Monographs. 2012;2012(44):49-55.

47. Gorin SS, Badr H, Krebs P, Das IP. Multilevel interventions and racial/ ethnic health disparities. JNCI Monographs. 2012;2012(44):100-11.

48. Frisell J, Lidbrink E, Hellstrom L, Rutqvist LE. Followup after 11 yearsupdate of mortality results in the Stockholm mammographic screening trial. Breast Cancer Res Treat. 1997;45(3):263-70.

49. McCarthy EP, Burns RB, Freund KM, et al. Mammography use, breast cancer stage at diagnosis, and survival among older women. J Am Geriatr Soc. 2000;48(10): 1226-33.

50. Petitti DB, Teutsch SM, Barton MB, Sawaya GF, Ockene JK, DeWitt T Update on the methods of the US Preventive Services Task Force: insufficient evidence. Ann Intern Med. 2009;150(3):199-205.

51. Schonberg MA, McCarthy EP. Mammography screening among women age 80 years and older: consider the risks. J Clin Oncol. 2009;27(4):640-1. author reply 641-642.

52. Anhang Price R, Zapka J, Edwards H, Taplin SH. Organizational factors and the cancer screening process. JNCI Monographs. 2010;2010(40):38-57.

53. Gorin SS. Prev practice in prim care. New York: Oxford University Press; 2014.

54. Yourman LC, Lee SJ, Schonberg MA, Widera EW, Smith AK. Prognostic indices for older adults: a systematic review. JAMA. 2012;307(2):182-92. 